\title{
Identification and characteristics of microRNAs from Bombyx mori Ping-an $\mathrm{He}^{\dagger 1,2}$, Zuoming Nie ${ }^{\dagger 1}$, Jianqing Chen1, Jian Chen ${ }^{1}$, Zhengbing Lv1, Qing Sheng1, Songping Zhou ${ }^{2}$, Xiaolian Gao ${ }^{1,3}$, Lingyin Kong ${ }^{1}$, Xiangfu Wu ${ }^{1}$, Yongfeng Jin*4 and Yaozhou Zhang*1
}

\begin{abstract}
Address: ${ }^{1}$ Institute of Biochemistry, Zhejiang Sci-Tech University, Hangzhou 310018, PR China, ${ }^{2}$ College of Science, Zhejiang Sci-Tech University, Hangzhou 310018, PR China, ${ }^{3}$ Department of Biology and Biochemistry, University of Houston, Houston, Texas 77204-5001, USA and ${ }^{4}$ Institute of Biochemistry, Zhejiang University, Hangzhou 310029, PR China

Email: Ping-an He - pinganhe@yahoo.com.cn; Zuoming Nie - wuxinzm@126.com; Jianqing Chen - cjqgqj@126.com; Jian Chen - chj1999@126.com; Zhengbing Lv - zhengbingl@yahoo.com.cn; Qing Sheng - csheng@zstu.edu.cn; Songping Zhou - szhou@zjip.com; Xiaolian Gao - xgao@uh.edu; Lingyin Kong - lingyin80@163.com; Xiangfu Wu - xfwu@sunm.shcnc.ac.cn; Yongfeng Jin* - jinyf@zju.edu.cn; Yaozhou Zhang* - yaozhou@zstu.edu.cn

* Corresponding authors †Equal contributors
\end{abstract}

Published: 28 May 2008

BMC Genomics 2008, 9:248 doi:I0.1 |86/|47|-2164-9-248
Received: 10 March 2008

Accepted: 28 May 2008

This article is available from: http://www.biomedcentral.com/I47I-2/64/9/248

(c) $2008 \mathrm{He}$ et al; licensee BioMed Central Ltd.

This is an Open Access article distributed under the terms of the Creative Commons Attribution License (http://creativecommons.org/licenses/by/2.0), which permits unrestricted use, distribution, and reproduction in any medium, provided the original work is properly cited.

\begin{abstract}
Background: MicroRNAs (miRNAs) are small RNA molecules that regulate gene expression by targeting messenger RNAs (mRNAs) and causing mRNA cleavage or translation blockage. Of the 355 Arthropod miRNAs that have been identified, only 21 are B. mori miRNAs that were predicted computationally; of these, only let-7 has been confirmed by Northern blotting.
\end{abstract}

Results: Combining a computational method based on sequence homology searches with experimental identification based on microarray assays and Northern blotting, we identified 46 miRNAs, an additional 21 plausible miRNAs, and a novel small RNA in B. mori. The latter, bmo-miR-100-like, was identified using the known miRNA aga-miR-100 as a probe; bmo-miR-100-like was detected by microarray assay and Northern blotting, but its precursor sequences did not fold into a hairpin structure. Among these identified miRNAs, we found 12 pairs of miRNAs and miRNA*s. Northern blotting revealed that some B. mori miRNA genes were expressed only during specific stages, indicating that B. mori miRNA genes (e.g., bmo-miR-277) have developmentally regulated patterns of expression. We identified two miRNA gene clusters in the $B$. mori genome. bmo-miR-2b, which is found in the gene cluster bmo-miR-2a-I/bmo-miR-2a- $1 * / b m o-m i R-2 a-2 / b m o-m i R-2 b / b m o-m i R-13 a * / b m o-m i R-/ 3 b$, encodes a newly identified member of the mir-2 family. Moreover, we found that methylation can increase the sensitivity of a DNA probe used to detect a miRNA by Northern blotting. Functional analysis revealed that II miRNAs may regulate I 3 B. mori orthologs of the 25 known Drosophila miRNA-targeted genes according to the functional conservation. We predicted the binding sites on the 167I 3'UTR of $B$. mori genes; 547 targeted genes, including 986 target sites, were predicted. Of these target sites, 338 had perfect base pairing to the seed region of 43 miRNAs. From the predicted genes, 61 genes, each of them with multiple predicted target sites, should be considered excellent candidates for future functional studies. Biological classification of predicted miRNA targets showed that "binding", "catalytic activity" and "physiological process" were over-represented for the predicted genes.

Conclusion: Combining computational predictions with microarray assays, we identified 46 B. mori miRNAs, 13 of which were miRNA*s. We identified a novel small RNA and 21 plausible B. mori miRNAs that could not be located in the available B. mori genome, but which could be detected by microarray. Thirteen and 547 target genes were predicted according to the functional conservation and binding sites, respectively. Identification of miRNAs in B. mori, particularly those that are developmentally regulated, provides a foundation for subsequent functional studies. 


\section{Background}

MicroRNAs (miRNAs) are a class of endogenous, small, non-coding, single-stranded RNA. They comprise approximately 19-25 nucleotides that are embedded within the stem regions of hairpin transcripts (called "pre-miRNAs") [1-9]. The first miRNA genes, lin-4 [5] and let-7 [6], were identified in Caenorhabditis elegans about a decade ago. Many endogenously encoded miRNAs have been detected in mammals, plants, insects, worms, and viruses through cloning, Northern blotting, microarray assay, sequencing of short RNA molecules [1-9], and computation [10-20]. By December 2007, 5,395 hairpin sequence entries, containing 5,234 mature miRNAs, have been stored in miRBase [21-23]. Sequence analyses have shown that some mature miRNAs are phylogenetically conserved, particularly in the first 8 residues at the $5^{\prime}$ end in species of the same kingdom (let-7 miRNAs are present in the human, mouse, rat, cow, dog, pig, chimpanzee, monkey, fish, and various insects, including Bombyx mori (B. mori)) [21]. In rare cases, mature miRNA sequences are conserved between animals and plants. For instance, mir-854, which is conserved in C. elegans, mouse, and human, has also been identified in plants [24]. However, hairpin sequences of precursor miRNAs are phylogenetically diverse. In addition to these characteristics, the genomic locations of miRNA precursor genes and the folding structures of miRNAs have been used to identify previously unknown miRNAs [10-20]. Lai et al. (2003) [11] stated that three characteristics allow miRNA genes to be identified using computational approaches: (i) miRNAs are generally derived from 70-100 nucleotide precursor transcripts having an extended stem-loop structure; (ii) miRNAs are usually conserved between genomes of related species; and (iii) miRNAs display a characteristic pattern of evolutionary divergence [10-20]. Additionally, genomic mapping of known miRNAs has enabled identification of orthologous miRNAs in other species (e.g., $B$. mori) where genomic annotations are lacking $[25,26]$.

miRNAs are well-studied RNA interference (RNAi) molecules that silence gene expression of sequence-specific targeted messenger RNAs (mRNAs) in all species in which they have been found [27-29]. miRNAs silence gene expression by inducing miRNA-guided mRNA degradation, or by inhibiting translation by forming a miRNAprotein complex that binds to targeted mRNAs [1-4]. Some miRNAs target the non-coding region of a RNA gene transcript [29]. miRNAs are a class of small RNAs that have temporal and spatial specific expression patterns, and that have important roles in the development, proliferation, differentiation, transformation, and death of cells [30-33]. miRNAs affect cell fate during all stages of life, from renewable, pluripotent embryonic cells to fully differentiated cells; miRNAs can also affect aberrant cell growth in some tumors and carcinomas [32]. The ability of miRNAs to silence gene expression through a post-transcriptional mechanism has promoted their use as genetic tools in basic mechanistic studies; miRNAs are also being developed as biomarkers, therapeutic targets and therapeutic agents.

Based on computational cross-genome comparison predictions and experimental identifications, over 5,000 miRNAs have been identified in various organisms, including 152 miRNAs from Drosophila melanogaster, 73 from Drosophila pseudoobscura, 54 from Apis mellifera, 45 from Anopheles gambiae, 21 from B. mori, 137 from C. elegans, and 541 human miRNAs (miRBase release 10.1, December 2007). B. mori is a species of Arthropod, closely related to Nematodes and Platyhelminthes. MiRBase contains 355 miRNA genes in Arthropods (mosquito, honey bee and fruit flies), 232 in Nematodes (C. elegans and C. briggsae) and 63 in Platyhelminthes (Schmidtea mediterranea) [34]. Of the 355 Arthropod miRNAs identified, 21 are B. mori miRNAs that were predicted computationally by our research group [19]; of these, only let-7 has been confirmed by Northern blotting $[35,36]$.

In this study, we report the first set of $B$. mori miRNA genes from larva, moth and pupa. We established our initial computational method for predicting miRNAs by genome-wide miRNA mapping using the available B. mori genomic sequences and Arthropod miRNAs. We further confirmed their identities using comprehensive microarray profiling and Northern blotting. miRNA targeted genes were predicted according to functional conservation and binding sites. Biological classification of predicted targets was determined by GO analysis.

\section{Results \\ Prediction of B. mori miRNAs}

Based on the sequences of all miRNAs previously identified in Arthropoda, we searched the B. mori genome by BLAST. According to the four criteria described in Materials and Methods, we identified 31 potential B. mori miRNAs and their precursor sequences. Information on predicted B. mori miRNAs, including names, lengths, source, and genomic position, are listed in Table 1. The length of the 31 predicted $B$. mori miRNAs ranged from 19 $\mathrm{nt}$ to $26 \mathrm{nt}$. We used the $m$ fold program to fold each of the 31 predicted $B$. mori pre-miRNA sequences, ranging in length from $70 \mathrm{nt}$ to $102 \mathrm{nt}$, and allowing G-U pairing, into hairpin structures [37]. The free energy of folding for these hairpin structures ranged from $-24.3 \mathrm{kcal} / \mathrm{mol}$ to $48.4 \mathrm{kcal} / \mathrm{mol}$.

\section{miRNA microarray assay}

Using the predicted B. mori miRNAs as guides, we designed probes to hybridize to targets in total RNA isolated from B. mori pupae and moths. Probes were classi- 
Table I: Information for all 46 predicted B. mori miRNA sequences

\begin{tabular}{|c|c|c|c|c|c|c|c|c|c|}
\hline miRNAs & Sequence & $\begin{array}{l}\text { Size } \\
\text { (nts) }\end{array}$ & $\begin{array}{c}\underset{\text { miRBasel }}{\text { first }} \\
\text { prediction }\end{array}$ & $\begin{array}{l}\text { Predicted } \\
\text { in this } \\
\text { study }\end{array}$ & $\begin{array}{c}\text { Northern } \\
\text { blot } \\
\text { identification }\end{array}$ & Source & Position & $\begin{array}{l}\text { Number of } \\
\text { mismatched } \\
\text { bp with } \\
\text { Drosophila } \\
\text { orthologs }\end{array}$ & $\begin{array}{c}\text { Number of } \\
\text { potential } \\
\text { targets }\end{array}$ \\
\hline bmo-let-7a & GAGGUAGUAGGUUGUAUAGU & 20 & yes & yes & yes & BAAB0103002I & $359-379$ & 0 & 10 \\
\hline bmo-let-7a* & ACUGUAUAGCCUGCUAACUUU & 21 & no & no & no & BAAB0103002I & $404-424$ & & 9 \\
\hline bmo-miR-I & UGGAAUGUAAAGAAGUAUGGAG & 22 & yes & yes & yes & BAAB0II0521I & $2140-2161$ & 0 & I \\
\hline bmo-miR-I* & UUCCGUGCUUCCUUACUUCCCA & 22 & no & no & no & BAAB0II0521I & $2098-2124$ & & 12 \\
\hline bmo-miR-2a-I & UAUCACAGCCAGCUUUGAUGAGC & 23 & no & yes & yes & BAAB0I090954 & $2892-2870$ & 0 & 22 \\
\hline bmo-miR-2a-l* & GGCAUCAAAGUCGGUUUGUCAUA & 23 & no & no & no & BAAB0I090954 & 2929-2907 & & 15 \\
\hline bmo-miR-2a-2 & UAUCACAGCCAGCUUUGAUGAGC & 23 & no & no & yes & BAAB0I 090954 & $2476-2454$ & 0 & 22 \\
\hline bmo-miR-2b & UAUCACAGCCAGCUUUGUUGAGU & 22 & no & yes & yes & BAAB0I090954 & $2355-2334$ & 2 & 38 \\
\hline bmo-miR-7 & GUAUGGAAGACUAGUGAUUUUGUUGU & 26 & yes & yes & no & BAAB0II89120 & $2066-2091$ & 0 & 6 \\
\hline bmo-miR-8 & UAAUACUGUCAGGUAAAGAUGUC & 23 & yes & yes & yes & BAAB0II75925 & $75-53$ & 0 & 5 \\
\hline bmo-miR-8* & GCAUCUUACCGGGCAGCAUUA & 21 & no & no & yes & BAAB0I 175925 & $115-95$ & & 16 \\
\hline bmo-miR-9 & UCUUUGGUUAUCUAGCUGUAUGA & 22 & yes & yes & yes & BAABOII 40220 & $35-56$ & 0 & 10 \\
\hline bmo-miR-9* & UCAUAAAGCUAGGUUACCGGAG & 22 & no & no & yes & BAABOII 140220 & $69-90$ & & 6 \\
\hline bmo-miR-10 & ACCCUGUAGAUCCGAAUUUGU & 21 & yes & yes & no & BAAB0II 187436 & $558-538$ & 1 & 3 \\
\hline bmo-miR-10* & ACAAAUUCGGUUCUAGAGAGGU & 22 & no & no & no & BAAB0II 187436 & $524-503$ & & 6 \\
\hline bmo-miR-13a* & CCUGUCAAAGCGGCGGUGAAA & 21 & no & no & no & BAABO 1090954 & $2777-2757$ & & 35 \\
\hline bmo-miR-13b & UAUCACAGCCAUUUUUGACGAGU & 23 & no & yes & yes & BAAB01090954 & $2615-2593$ & 1 & 6 \\
\hline bmo-miR-14 & UCAGUCUUUUUCUCUCUCCUA & 21 & yes & no & yes & BAABOI 082886 & $457-437$ & 0 & 8 \\
\hline bmo-miR-3I & GGCAAGAAGUCGGCAUAGCUG & 21 & yes & yes & yes & BAABOI 205265 & $3219-3239$ & 3 & 14 \\
\hline bmo-miR-34 & GGCAGUGUGGUUAGCUGGUUGUGUA & 25 & yes & yes & no & BAAB0II 47222 & $|437-| 47 \mid$ & I & 197 \\
\hline bmo-miR-46 & UGUCAUGGAGUUGCUCUCUUUA & 22 & no & no & no & BAAB01025185 & $703-722$ & 2 & 7 \\
\hline bmo-miR-46* & UGAAGAGAGCUAUCCGUCGACA & 22 & no & no & no & BAAB01025185 & $664-685$ & & 10 \\
\hline bmo-miR-7I & UGAAAGACAUGGGUAGUGA & 19 & yes & no & no & BAAB0 1131802 & $2236-2218$ & 0 & 6 \\
\hline bmo-miR-79 & UUCAUAAAGCUAGAUUACCAAAGCAU & 26 & no & yes & no & BAABOIII 3362 & $535-510$ & I & 2 \\
\hline bmo-miR-87 & UGAGCAAACUUUCAGGUGUGU & 21 & no & yes & no & BAAB0II 64262 & $758-778$ & 2 & 4 \\
\hline bmo-miR-133 & UUGGUCCCCUUCAACCAGCUGU & 22 & no & yes & no & BAAB0I 208622 & $2993-3014$ & 0 & 11 \\
\hline bmo-miR-184 & ACUGGACGGAGAACUGAUAAGGGC & 24 & no & yes & no & BAABOI083504 & $|684-166|$ & 0 & 7 \\
\hline bmo-miR-2 10 & UUGUGCGUGUGACAGCGGCU & 20 & no & yes & no & BAAB0 1031372 & $2309-2290$ & 0 & 95 \\
\hline bmo-miR-263a & AAUGGCACUGGAAGAAUUCAC & 21 & yes & yes & yes & BAAB0II 06279 & $2833-2853$ & 2 & 4 \\
\hline bmo-miR-263b & CUUGGCACUGGGAGAAUUCACAG & 23 & yes & yes & no & BAABOIOI 5424 & $804-782$ & 0 & 19 \\
\hline bmo-miR-263b* & CGUGAAUUUCCCGAUGCCUUA & 21 & no & no & no & BAABOI0I5424 & $764-744$ & & 8 \\
\hline bmo-miR-275 & AGUCAGGUACCUGAAGUAGCGCGCG & 25 & yes & yes & no & BAAB0III 8959 & $480-456$ & 0 & 41 \\
\hline bmo-miR-276a & UAGGAACUUCAUACCGUGCUCU & 22 & no & yes & yes & BAAB0II 99848 & $358-337$ & 0 & 1 \\
\hline bmo-miR-276a* & (C)AGCGAGGUAUAGAGUUCCUA(CG) & 22 & yes & no & no & BAABOI 199848 & $395-374$ & & 6 \\
\hline bmo-miR-277 & UAAAUGCACUAUCUGGUACGACA & 23 & yes & no & yes & BAABOI014298 & $12099-12121$ & 0 & 4 \\
\hline bmo-miR-279 & UGACUAGAUCCACACUCAU & 19 & yes & yes & yes & BAAB01102170 & $714-732$ & 3 & 0 \\
\hline bmo-miR-279* & AUGAGUGGAGGUUUAGUGCA & 20 & no & no & no & BAAB0II02170 & $679-698$ & & 37 \\
\hline bmo-miR-28Ia & ACUGUCAUGGAGUUGCUCUCUU & 22 & no & yes & yes & BAAB01025185 & $701-722$ & 3 & 18 \\
\hline bmo-miR-28Ia* & AAGAGAGCUAUCCGUCGACAGU & 22 & no & no & yes & BAABO 1025185 & $666-687$ & 0 & 10 \\
\hline bmo-miR-282 & UAGCCUCUCCUUGGCUUUGUCUG & 23 & yes & yes & no & BAAB01005896 & $688-710$ & 4 & 32 \\
\hline bmo-miR-283 & UAAAUAUCAGCUGGUAAUUCUGGG & 24 & yes & yes & no & BAAB0I 105947 & $466-443$ & 0 & 4 \\
\hline bmo-miR-305 & UGUACUUCAUCAGGUGCUCUGG & 22 & Yes & yes & no & BAAB0III 8959 & $386-365$ & 0 & 16 \\
\hline bmo-miR-305* & CAGGCGCUUGUUGGAGUACACU & 22 & no & no & no & BAAB0IIII8959 & $352-331$ & & 66 \\
\hline bmo-miR-307 & CAUCACAACCUCCUUGAGUGAGCGAU & 26 & yes & yes & no & BAAB0II 78566 & $1478-1503$ & 0 & 19 \\
\hline bmo-miR-3I7 & AGUGAACACAGCUGGUGGUAUC & 22 & no & yes & no & BAABOII 40584 & $492-471$ & I & 21 \\
\hline bmo-bantam & UGAGAUCAUUGUGAAAGCUAAUU & 23 & no & no & yes & BAAB01089624 & $1355-1373$ & 1 & 1 \\
\hline bmo-miR-100-like & AACCCGUAGAUCCGAACUUGUG & 22 & no & no & yes & BAABOII29141 & $1755-1776$ & 0 & 5 \\
\hline
\end{tabular}

fied into one of three groups, i.e., those designed to detect: 1) the 31 predicted miRNAs and their opposite-strand miRNAs; 2) the 704 known miRNAs in miRBase, which consist of miRNAs from several species, including six worm species, D. melanogaster (78 probes), D. pseudoobscura (4 probes), A. gambiae (14 probes), A. mellifera (7 probes), C. elegans (114 probes), C. briggsae (39 probes), and human (448 probes); and 3 ) control sequences, including the $5 \mathrm{~S}$ rRNA of $B$. mori and $D$. melanogaster. The chip contained 954 probes in total. Each probe was repeated three times on the chip to ensure assay reproducibility.

Microarray results are shown in Figure 1. Comparison of the signal strengths for the miRNAs detected by microarray assays of RNA isolated from B. mori pupa and moth stages are shown in Figure 2. In most cases, results between spots for the same miRNA genes were very consistent in RNA isolated from B. mori pupa and moth $(\mathrm{k}=$ $0.908, b=0.3104, R^{2}=0.8025$ ). However, expression levels of some miRNAs exhibited obvious differences between the two developmental stages. Sequences exhibiting signal strengths of $>80$ were considered to be detected miRNA sequences. We detected 114 small RNAs from total RNA isolated from B. mori pupae and moths based on this cutoff. We applied a more stringent analysis by excluding miRNAs differing by only one single base in their sequences or by two bases near sequence ends, and identified 67 miRNAs and a novel small RNA. Of these 67 detected miRNAs, 27 miRNAs belonged to the 31 predicted miRNAs and 12 miRNAs were miRNA*s; another 28 miRNAs were detected only in the microarray assay 

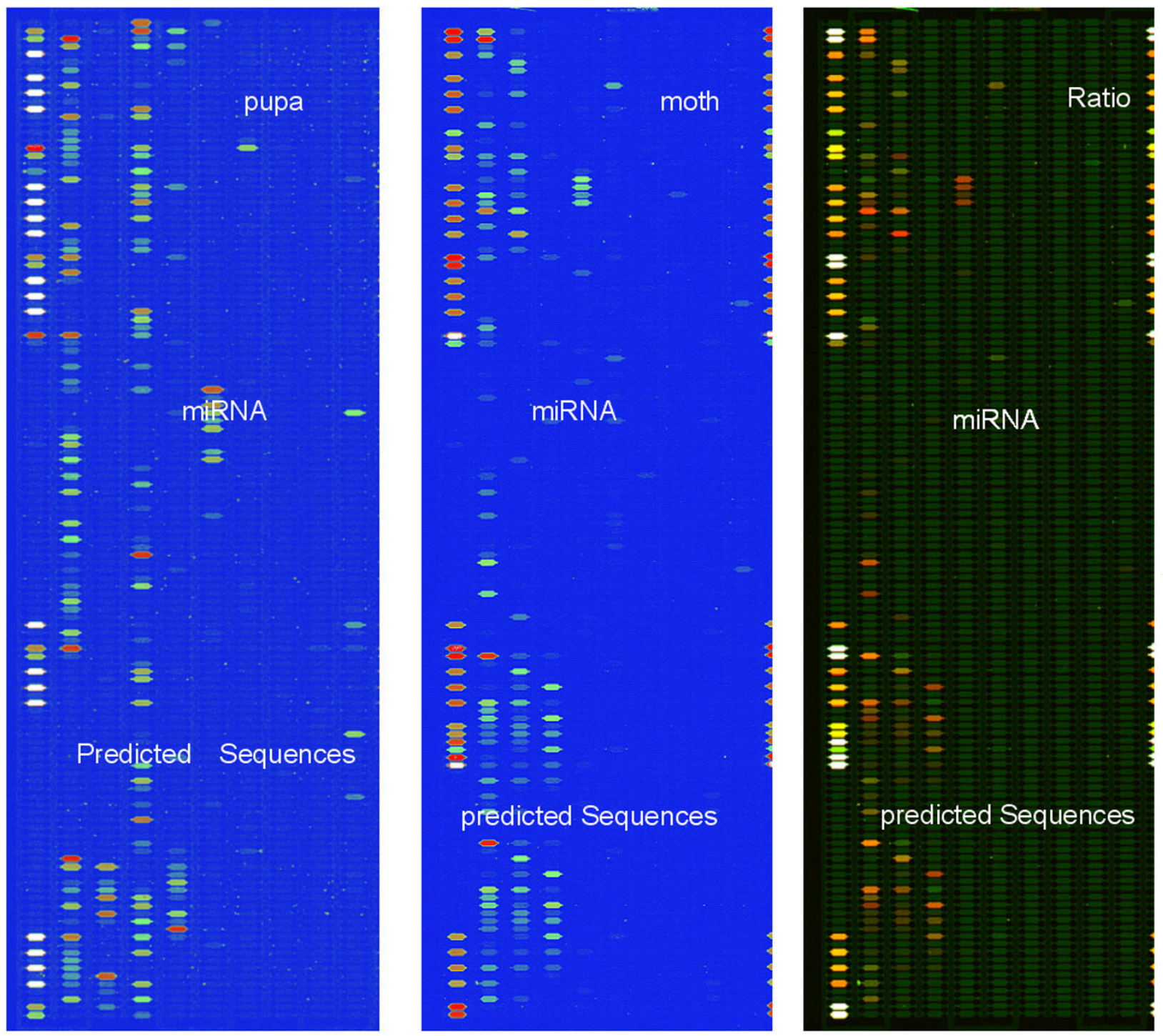

\section{Figure I}

miRNA chip hybridization in B. mori pupa and moth.

using probes designed to detect known miRNA from other species. We used these 28 miRNA sequences as query sequences to perform BLAST searches against the $B$. mori genome. Seven of these sequences were identified in the $B$. mori genome and their precursor sequences could be folded into hairpin structures and, as such, these seven sequences were likely to be $B$. mori miRNAs. We designated these sequences bmo-miR-13a*, bmo-miR-14, bmomiR-46, bmo-miR-46* , bmo-miR-71, bmo-miR-277 and bmobantam. Although bmo-miR-13a was one of the 31 miRNAs that were predicted, it was not detected by microarray assay, but its opposite-strand miRNA, named bmo-miR$13 a^{*}$, was detected. The remaining 21 miRNAs were detected by microarray assay, but were not found in the available B. mori genome (possibly because sequencing of the $B$. mori genome is incomplete). We considered these 21 miRNAs to be plausible B. mori miRNAs. A novel small RNA was found using a probe designed to detect the known miRNA aga-miR-100. This small RNA was detected by microarray and Northern blotting (Figure 3A) and was identified in the $B$. mori genome, but its precursor sequence failed to fold into a hairpin structure. This novel small RNA was designated bmo-miR-100-like.

In conclusion, combining computational predictions with microarray assays, we identified 46 B. mori miRNAs, 
(A)

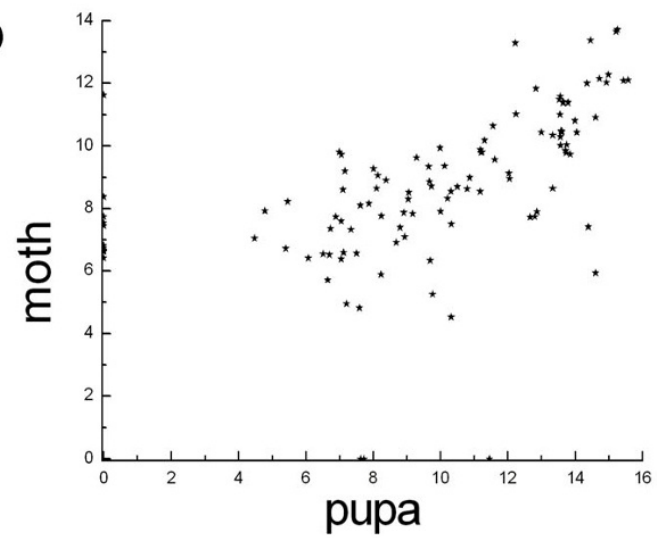

(B)

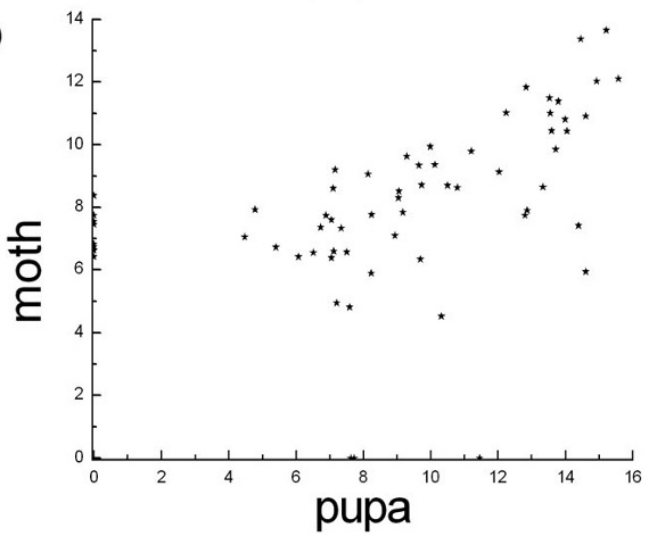

(C)

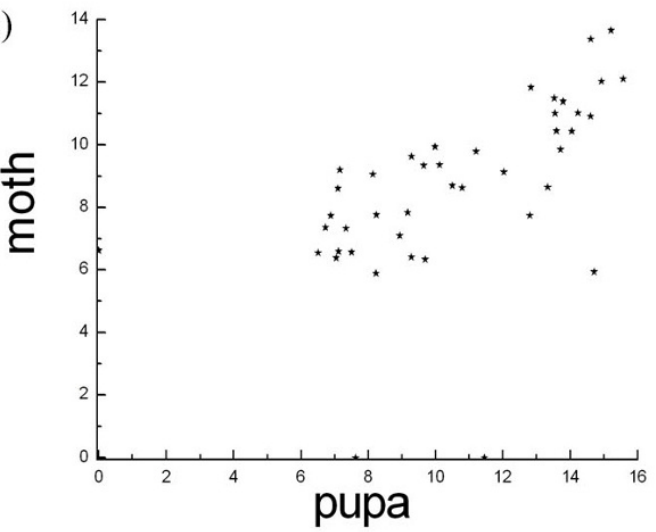

Figure 2

Signal strength of detected miRNAs by microarray assay in B. mori pupa and moth. a. all detected miRNAs; b. removed redundant miRNAs; c. B. mori miRNAs. $N$ depicts the signal strength of detected miRNAs.

13 of which were miRNA*s. We identified a novel small RNA and 21 plausible B. mori miRNAs that could not be found in the available B. mori genome, but which was detected by microarray. Detailed information for these 46 miRNAs and the novel small RNA are listed in Table 1. The 21 plausible B. mori miRNAs are listed in Table 1 of Additional file 1.

\section{Identification and determination of development-specific expression patterns of B. mori miRNAs by Northern blotting}

Northern blotting was done based on microarray assay results to confirm the expression of $B$. mori miRNAs. Expression of the 46 identified miRNAs, the novel small RNA, and another 21 plausible miRNAs was investigated by Northern blotting of small-sized RNAs isolated from the larva, pupa, and moth stages of B. mori. Twenty-six miRNAs were stably expressed in $B$. mori in at least one developmental stage (Figure 3 ). Of these 26 miRNAs, 18 belong to the group of 46 miRNAs identified by microarray assay (Figure 3A) and 7 belong to the group of 21 plausible B. mori miRNAs (Figure $3 \mathrm{~B}$ ); the novel small RNA bmo-miR-100-like is shown in Figure 3A.

Development-specific expression patterns for some $B$. mori miRNAs were determined by Northern blotting. Some $B$. mori miRNA genes were expressed only in certain stages (Figure 3). bmo-miR-1, bmo-let-7a, bmo-miR-8, bmomiR-14, bmo-miR-276a, bmo-miR-279 were strongly expressed in all developmental stages (larva, pupa and moth). They were uniformly expressed, suggesting that these miRNAs may play an important role in the regulation of some constitutive process in B. mori. Of these miRNAs, bmo-miR-8 has an opposite-strand miRNA, bmo-miR$8^{*}$, which was detected by Northern blotting (Figure 3A) at very low levels in larva, pupa and moth. bmo-let-7b, bmo-let-7c, bmo-miR-9, bmo-miR-9*, bmo-miR-100-like, bmo-miR-263a, bmo-miR-31 and bmo-bantam were expressed in larva and pupa, but were not detected in moth; of these miRNAs, bmo-miR-9 and bmo-miR-9* are also complementary miRNAs. bmo-miR-281a, bmo-miR$281 a^{*}, b m o-m i R-281 b^{*}, b m o-m i R-13 b$ and bmo-miR- $2 b$ were expressed most strongly in larva, although they were also expressed in pupa and moth. bmo-mri-2a, bmo-miR$100, b m o-m i R-276 b$ and bmo-let-7d were also expressed in larva, pupa and moth; bmo-mri-2a and bmo-miR-100 were expressed most strongly in pupa; bmo-miR-276b was expressed most strongly in moth; bmo-let-7d was expressed most weakly in moth. bmo-miR-277 was expressed only in moth and not detected in larva and pupa; the precursor of bmo-miR-277 was also detected [see Figure S1 of Additional file 1]. Similarly, bmo-miR-289 was expressed weakly only in pupa and was not detected in larva and moth. Expression of a miRNA in a specific developmental stage may suggest a role for it in the developmental process.

\section{Computational prediction of B. mori miRNA targets}

In the TarBase database, there are 23 miRNAs that regulate 34 targeted genes in D. elanogaster. miRNA functions could be evolutionally conserved between species such as $B$. mori and D. melanogaster [38]. To deduce the function of $B$. mori miRNAs, we searched for targeted genes of their 


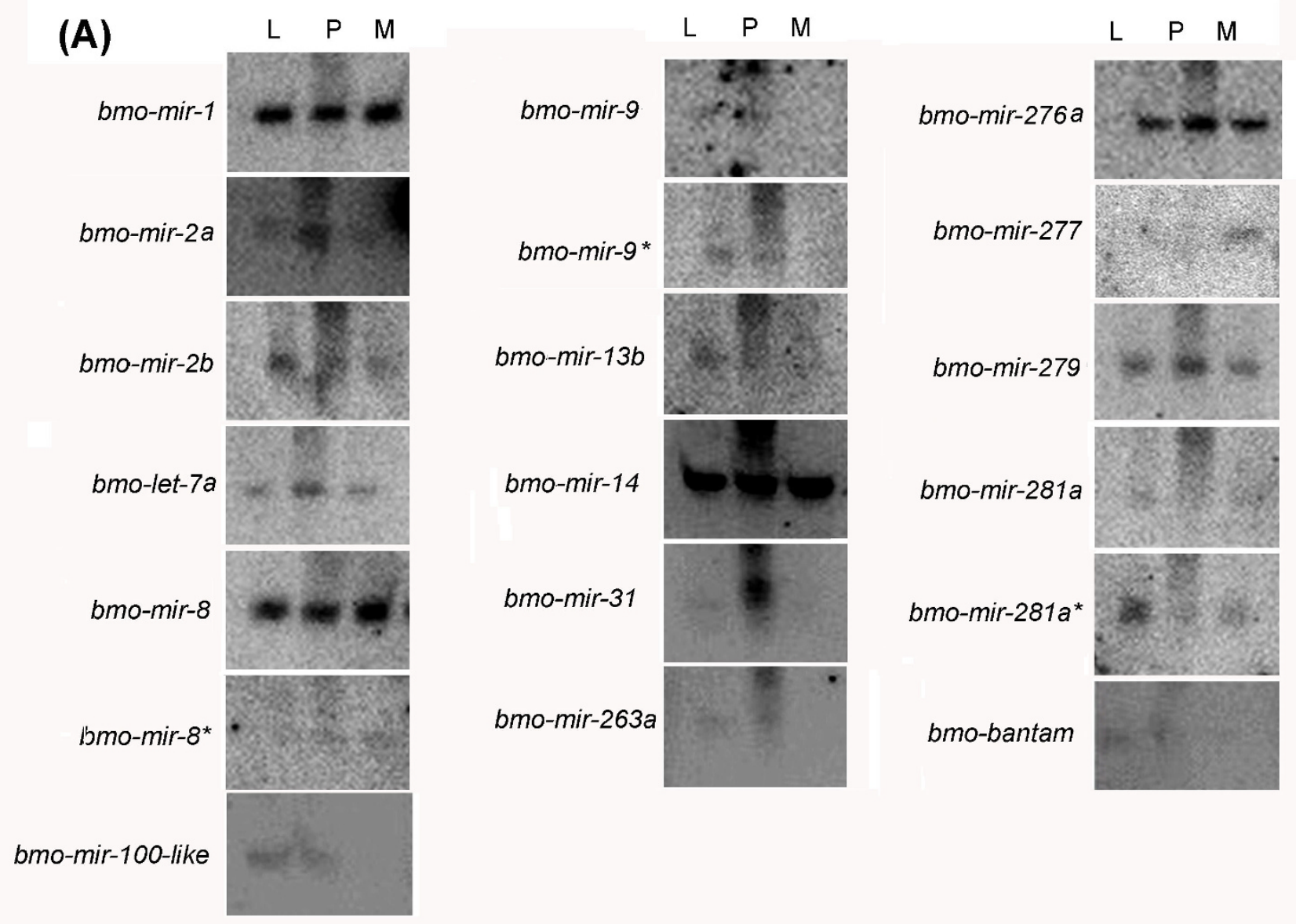

\section{(B)}

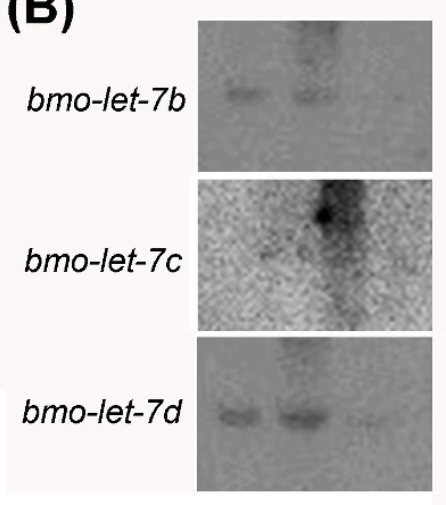

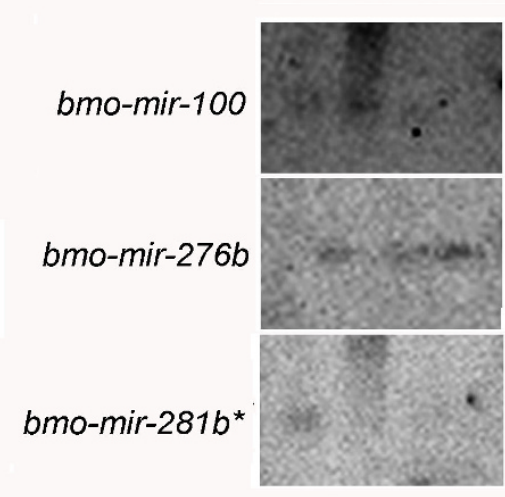

bmo-mir-289

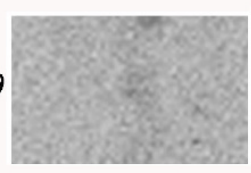

S rRNA $=$

\section{Figure 3}

Identification of miRNAs by Northern blotting using DNA probes complementary to the miRNA sequences. (A). Northern blotting analysis of the miRNAs that belong to the group of 46 miRNAs identified by microarray assay, including the novel small RNA bmo-miR-100-like. (B). Northern blotting analysis of the miRNAs that belong to the group of 21 plausible B. mori miRNAs. The $5 S$ rRNA band was the loading control. L, larva; P, pupa; M, moth. 
orthologous miRNAs reported in D. melanogaster in the TarBase database [39]. In the 46 B. mori miRNAs identified, we found 11 miRNAs, belonging to 8 miRNA families; Drosophila orthologs of these 11 miRNAs have been reported in TarBase and are known to regulate the expression of at least 25 genes. These 11 miRNAs may regulate $13 \mathrm{~B}$. mori orthologs of the 25 Drosophila miRNA-targeted genes according to binding [see Additional file 2]. BmomiR-133 may regulate two B. mori orthologs of Mus musculus miRNA targeted genes, SRF and Ptbp2 because of the perfect binding between miRNAs and the complementary sites. GO analysis showed that "nucleus" was over-represented for Drosophila orthologs of the 13 potential targeted genes.

We calculated the potential binding sites between miRNAs and the 3'UTR of mRNAs to determine the potential miRNA targeted genes more globally. With settings hybrid 11 and hybridf22 described in Materials and Methods, we obtained 465 and 262 targeted genes, respectively. One hundred and eighty genes were involved in the two settings simultaneously. Removing redundancy, 547 targeted genes including 986 target sites were predicted. Of these binding sites, 338 had perfect base pairing to the seed region of 43 miRNAs. Of the 46 identified miRNAs, bmo-miR-279 was the only one for which we failed to find target sites. Certain miRNAs may have more than one target, and some predicted targets may be regulated by more than one miRNA [see Additional file 3 and Table 1]. Additionally, we found 61 3'UTRs that each contain multiple potential binding sites to a single miRNA [see Additional file 3]; these miRNA-mRNA duplexes showed higher specificity than others. Grun et al. (2005) predicted that at least $15 \%$ of $D$. melanogaster genes were regulated by at least one known miRNA [38]. Using settings hybrid11 (hybridf22), we found that $28 \%(16 \%)$ of annotated 1671 3'UTR sequences (corresponding to 1671 genes) had at least one binding site to miRNAs identified in this study. Compared with hybrid11, hybridf22 showed a significantly higher specificity. Biological classification of predicted miRNA targets was done by GO analysis to obtain insight into the function of B. mori miRNAs. GO analysis was completed by submitting gene sequences online (see Materials and Methods). In the predicted targets with settings hybrid11 (hybridf22), 360 (214) genes were assigned to the GO terms of "molecular function" ontology by BLAST, "binding" and "catalytic activity" of which over-represented for the 360 (214) genes; in the "biological process" ontology, "physiological process" over-represented among the 277(184) genes which were predicted with setting hybrid11 (hybridf22) and assigned to GO terms by BLAST [see Additional file 3].

\section{Discussion}

We identified 46 miRNAs from B. mori using computational analyses and microarray assays. Of these 46 putative miRNAs, 18 were also confirmed by Northern blotting (Figure 3); although the remaining miRNAs were not detected by Northern blotting, they were considered to be miRNAs because they were detected by microarray and their precursors could adopt a hairpin structure. The reason why some miRNAs could not be detected by Northern blotting may be due to expression below the detection limit, or the very narrow temporal expression window for these miRNAs.

Methylated RNA probes have been employed by some research groups for RNA detection [40-42]. We found that methylation of the DNA probe used to detect miRNA by Northern blotting could increase sensitivity. Using methylated DNA probes, signal strength was significantly improved in Northern blots for bmo-miR-14 and bmo-miR$8^{*}$, which could not be detected using non-methylated DNA probes [see Figure S2 of Additional file 1]. Improvement may be due to the effect of methylation on base pairing of the probe with the target, or on the secondary structure of the probe.

\section{Analysis of B. mori miRNAs clusters}

miRNA clusters have been reported in many species. A cluster usually contains two or three miRNA genes, although larger clusters have also been identified, such as the six-member $h s a-m i R-17$ cluster $[7,43,44]$ or the D. melanogaster cluster containing eight miRNA genes [45]. Clustered miRNA genes can share a high degree of similarity in nucleotide composition, but occasionally the miRNA sequences differ significantly $[30,46]$. Expression profiles for clustered genes are also very similar, raising the possibility that transcription of these miRNAs is controlled by a common regulatory element [47]. Examining the positions of the identified miRNAs in the B. mori genome, we identified two miRNA clusters (Figure 4): bmo-miR-2a-1/ bmo-miR-2a-1 */bmo-miR-2a-2/bmo-miR-2b/bmo-miR-13a*/ bmo-miR-13b; and bmo-miR-275/bmo-miR-305/bmo-miR$305^{*}$. The lengths of the two cluster sequences were 498 and $208 \mathrm{bp}$, respectively. Genes in the cluster containing bmo-miR-2a-1/bmo-miR-2a-1*/bmo-miR-2a-2/bmo-miR-2b/ bmo-miR-13a*/bmo-miR-13b are members of the mir-2 miRNA family. This cluster contains bmo-miR-2a, which can be derived from two different precursor miRNAs transcribed from two paralogous miRNA genes, $b m o-m i R-2 a-1$ and $b m o-m i R-2 a-2$. The opposite strand to bmo-miR-2a-1 also encodes a miRNA. Searching miRBase, we found that the bmo-miR-2a sequence was identical to the dme-miR-2a, $d p s-m i R-2 a$, ame-miR-2, and aga-miR-2 sequences, but we failed to identify any other miRNA that had the same sequence as $b m o-m i R-2 b$ (Figure 5); therefore, $b m o-m i R-2 b$ is a newly identified member of the mir-2 miRNA family. 

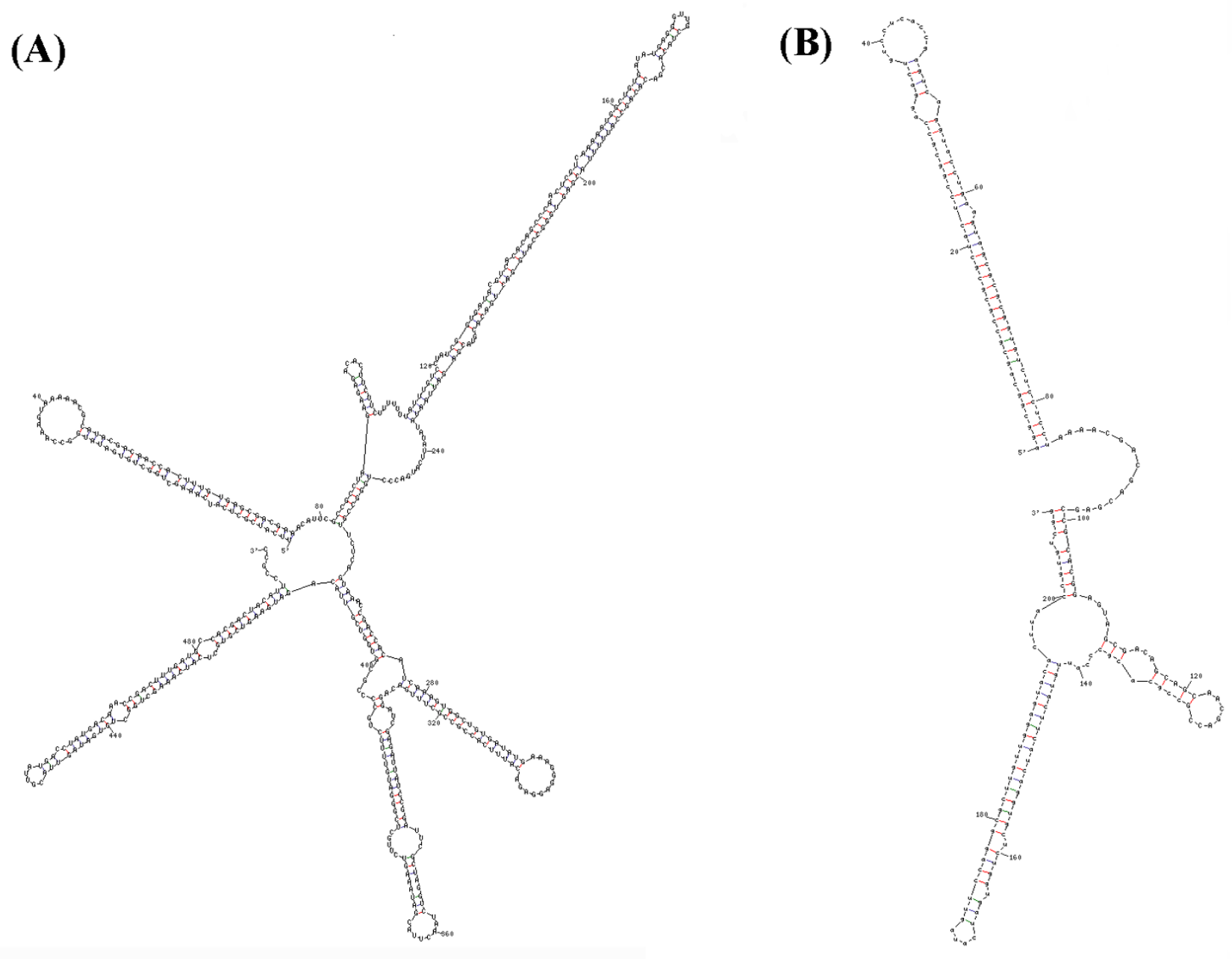

\section{Figure 4}

Secondary structures of miRNA clusters. a. bmo-miR-2a- //bmo-miR-2a- $/$ */bmo-miR-2a-2/bmo-miR-2b/bmo-miR- $/ 3 a * / b m o-$ miR-I 3b; b. bmo-miR-275/bmo-miR-305/bmo-miR-305*.

Searching the D. melanogaster and A. gambiae miRNAs, we found that corresponding mir-2 and mir-13 family members also assembled into clusters in these two species. mir2 and mir-13 repress translation of the target genes, grim, $s k l$ and $r p r$, suggesting that they may be involved in regulating apoptosis [48]. The cluster bmo-miR-275/bmo-miR$305 / b m o-m i R-305^{*}$ is also found in D. melanogaster, D. pseudoobscura and A. gambiae. bmo-miR-275 and bmo-miR305 belong to different miRNA families, mir-275 and mir305. The functions of these two miRNA families remain unknown.

\section{Analysis of B. mori miRNAs}

The identified 46 B. mori miRNAs belonged to 29 miRNA families based on sequence similarity (Table 2). Among the 46 identified miRNAs, we found 12 pairs of miRNAs and miRNA*s. Scanning miRBase, we determined that seven of these 12 miRNA*s had not been reported. These newly identified miRNAs are bmo-miR-2a*, bmo-miR- $8^{*}$, bmo-miR-13a*, bmo-miR-46*, bmo-miR-263*, bmo-miR279*, and bmo-miR-305*.

Observing the orthologous miRNA genes for each family in miRBase, we found that 15 of the 29 families are exclusive to Arthropoda; the other 12 families are found in many species, including five species of Arthropoda. miRNAs having the most orthologs are mir-133 and mir-9, which are found in 25 and 23 animal species, including D. melanogaster and C. elegans.

We determined the number of orthologous and paralogous genes for each detected miRNA. The let-7 miRNA 
(A)

\begin{tabular}{|c|c|}
\hline$b m o-m i r-2 b$ & JAUCACAGCCAGCUUUGUUGAGU \\
\hline bmo-mir-2a & UAUCACAGCCAGCUUUGAUG \\
\hline$d m e-m i r-2 a$ & UAUCACAGCCAGCUUUGAUG \\
\hline$d p s-m i r-2 a$ & UAUCACAGCCAGCUUUGAUG \\
\hline ame-mir-2 & UAUCACAGCCAGCUUUGAUC \\
\hline aga-mir-2 & UAUCACAGCC \\
\hline celmir-2 & UAUCACAGC \\
\hline$d m e-m i r-2 b$ & UAUCACAGCCAGCUUUGAGGAGC \\
\hline$d p s-m i r-2 b$ & UAUCACAGCCAGCUUUGAGGAGC \\
\hline dme-mir-2c & UAUCACAGCCAGCUUUGAUGGGC \\
\hline$d p s-m i r-2 c$ & UAUCACAGCCAGCUUUGAUGGGC \\
\hline
\end{tabular}

(B)

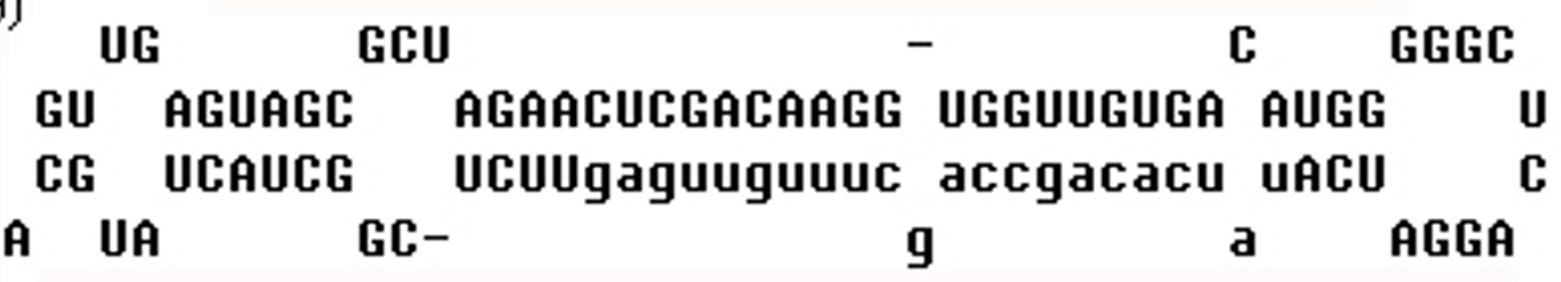

Figure 5

Homologous analysis of mir-2 family.bmo-miR-2b is considered to be a new member of the mir-2 family. (A), Multi-alignment of mir-2 family miRNAs. (B), Hairpin structure of the precursor of bmo-miR-2b.

family contains the most members; 77 let-7 family members were identified in miRBase [see Figure S3 of Additional file 1].

\section{Analysis of B. mori precursor miRNA sequences}

Variations in pre-miRNA sequences are related to their evolutionary history [22], so we compared the sequences of $B$. mori pre-miRNAs and members of the same family of pre-miRNAs in the miRBase database (Table 2). The number of the pre-miRNAs is greater than the number of mature miRNAs because mature miRNAs can be derived from two or more pre-miRNAs. This is consistent with the literature: mature miRNAs are conserved, but pre-miRNAs are diverse [23].

Members of the bantam, mir-71, mir-275, mir-277, mir-279 miRNA families have a high degree of similarity in their precursor sequences. bmo-miR-279 and other mir-279 precursor sequences have sequence similarities of $>55 \%$; bmo-miR-277 precursors share $>56 \%$ sequence similarity; bmo-miR-27 precursors share $>57 \%$ sequence similarity; bmo-miR-71 precursors share $>59 \%$ sequence similarity; and bmo-bantam precursors share $>60 \%$ sequence similarity.

Based on the results of our sequence comparisons, we determined the phylogenetic tree for each miRNA family using the DNAStar MegAlign program to analyze evolutionary relationships between miRNA members from each family. Many of the B. mori miRNA families were found only in Arthropoda; their mature and pre-miRNAs are highly conserved. For these miRNAs, we found that these $B$. mori miRNAs have close evolutionary relationships with A. mellifera, and A. gambiae miRNAs. We found that these $B$. mori miRNAs are located on the same branches of the phylogenetic tree as related miRNAs from D. melanogaster, D. pseudoobscura, A. mellifera, and A. gambiae. The phylogenetic tree for the mir-1 and mir-184 miRNA family is shown in Figure 6; other detailed results are listed in Additional file 4.

We compared the hairpin structures of B. mori miRNA precursor sequences to those of related D. melanogaster, $D$. pseudoobscura, A. mellifera, and A. gambiae miRNA precur- 
Table 2: Comparisons of sequence similarity between $B$. mori miRNAs and corresponding miRNA family members

\begin{tabular}{|c|c|c|c|c|c|}
\hline \multirow[t]{2}{*}{ Index } & \multirow[t]{2}{*}{ Family } & \multicolumn{2}{|c|}{ Mature } & \multicolumn{2}{|c|}{ Pre-miRNA } \\
\hline & & Count & Similarity & Count & Similarity \\
\hline I & bantam & 5 & $>91 \%$ a & 5 & $* * *$ \\
\hline 2 & let-7 & 72 & $>80 \%$ a & 101 & $*$ \\
\hline 3 & mir-I & 14 & $>86 \% \mathrm{a}$ & 17 & ** \\
\hline 4 & mir-2 & 17 & $>92 \% \mathrm{a}$ & 16 & $* *$ \\
\hline 5 & mir-7 & 25 & $>85 \%$ & 41 & $*$ \\
\hline 6 & mir-8 & 5 & $100 \%$ & 5 & $*$ \\
\hline 7 & mir-9 & 29 & $>83 \%$ a & 48 & \\
\hline 8 & mir- 10 & 30 & $>86 \% \mathrm{a}$ & 34 & $* *$ \\
\hline 9 & mir- 14 & 4 & $100 \%$ & 9 & $*$ \\
\hline 10 & mir-3I & 17 & $>81 \%$ & 18 & $* *$ \\
\hline II & mir-34 & 31 & $>80 \%$ a & 34 & $*$ \\
\hline 12 & mir-46 & 3 & $>90 \%$ & 3 & $*$ \\
\hline 13 & mir-7I & 3 & $100 \%$ & 3 & $* * *$ \\
\hline 14 & mir-79 & 6 & $>90 \%$ & 6 & $*$ \\
\hline 15 & mir-87 & 5 & $>92 \%$ & 5 & $* *$ \\
\hline 16 & mir- 133 & 33 & $>88 \%$ a & 37 & $*$ \\
\hline 17 & mir- 184 & 17 & $>90 \%$ a & 18 & ** \\
\hline 18 & mir-210 & 12 & $>90 \% \mathrm{a}$ & 12 & ** \\
\hline 19 & mir-263 & 8 & $>80 \%$ & 8 & $* *$ \\
\hline 20 & mir-275 & 4 & $100 \%$ & 4 & $* * *$ \\
\hline 21 & mir-276 & 6 & $>91 \%$ a & 7 & $*$ \\
\hline 22 & mir-277 & 5 & $100 \%$ & 5 & $* * *$ \\
\hline 23 & mir-279 & 4 & $100 \%$ & 4 & $* * *$ \\
\hline 24 & mir-28I & 5 & $>83 \%$ & 7 & $*$ \\
\hline 25 & mir-282 & 5 & $>85 \%$ & 5 & $*$ \\
\hline 26 & mir-283 & 4 & $100 \%$ & 4 & $* *$ \\
\hline 27 & mir-305 & 5 & $100 \%$ & 5 & ** \\
\hline 28 & mir-307 & 4 & $100 \%$ & 4 & $*$ \\
\hline 29 & mir-3/ 7 & 5 & $>92 \%$ a & 5 & $* *$ \\
\hline
\end{tabular}

family: the name of miRNA family; count: the number of miRNAs in other species in the same miRNA family; similarity: the similarity between the sequences of $B$. mori miRNAs and the sequences of other members of the same family; the letter "a" denotes that this miRNA is identical to other mature miRNAs in the family; * denotes that this miRNA is only highly conserved in the miRNA-encoding region; ** denotes that this miRNA is highly conserved in the miRNAencoding region and its opposite-strand region (miRNA*-encoding region); *** ** denotes that this miRNA is highly conserved in the precursor sequences.

sor sequences. Conserved regions of the precursors are found in two arms of the stem-loop structure. The results of comparisons between the hairpin structures of bmo$m i R-1$, aga-miR-1, ame-miR-1, dme-miR-1 and $d p s-m i R-1$ precursors are shown in Figure 7. Additional file 5 shows the secondary structures of $B$. mori miRNAs. These results strongly indicate that the identified $46 \mathrm{~B}$. mori sequences are miRNAs.

\section{Computational prediction of B. mori miRNA targets}

MiRNA can regulate the protein expression of genes based on the level of complementarity between miRNA seed sequences and binding sites on target mRNA [49-51]. For animals, the seed sequences of miRNA can bind to com- plementary sites on 3'UTR of its targeted gene. Complementary sites in targeted genes and seed sequences of miRNAs may be conserved in various species; this may result in the functional conservation of miRNAs, and the orthologous miRNAs may regulate orthologs of the targeted genes. The prediction for miRNA targeted genes according to functional conservation may offer additional insights into the function of miRNAs. Grun et al. (2005) computed the conserved regulatory microRNA-mRNA relationships [38]. They found that 50 unique gene pairs were predicted to be targeted by homologous microRNAs, including 60 microRNA-mRNA regulatory relationships [38]. Weaver et al. (2007) demonstrated that some microRNAs function in the same or similar way in Drosophila and bee (e.g., mir-9a may control sensory organ precursors (SOPs) between Drosophila and bee) [52]. Functional prediction based on cross-species conservation may provide a reference for studying on the functions of $B$. mori miRNAs. Based on this method, we identified 12 miRNAs that regulate 15 potential targeted genes [see Additional file 2]. Most Drosophila orthologs of these potential targeted genes were the vital genes for development, including transcriptional regulator, apoptosis-related genes and genes involved in signal transduction pathways. mir-2 controls HLHmdelta, and mir-7 controls HLHm3, HLHm5, HLHmgamma, M4 and TOM, in D. melanogaster; these six genes are involved in the Notch signaling pathway. B. mori homologs [GenBank: Bmo.1984 and Bmo.4220] of these six genes can bind perfectly to mir-2 (bmo-miR-2a, bmo-miR-2b, bmo-miR-13a* and bmo-miR$13 b$ ) and $b m o-m i R-7$, respectively. mir- 2 and mir-7 may be related to the Notch signaling pathway in $B$. mori; this hypothesis is consistent with previous predictions $[38,53]$. Cross-species comparisons allow for the identification of evolutionarily conserved and probable functional target sites. The 12 miRNAs could play parts in the development of $B$. mori by regulating their targeted genes, including the 15 potential targeted genes identified in this study.

We predicted many miRNA targeted genes by calculating target sites. Interestingly, we have studied B. mori profilin gene [GenBank: EF112402]; there was not a linear relationship between the transcription level of this gene and protein expression (data not shown). The long 3'UTR of this gene was found to have nine potential binding sites [see Additional file 3]. Profilin plays an important part in biologically active cellular compartments [54]. It regulates the polymerization, depolymerization, and dynamics of actin, which are important for determining cell shape and movement through the cytoplasm [54].

We can predict miRNA targets specifically according to the level of complementarity between the seed region of miRNA and 3'UTR of mRNA [49]. We set stringent set- 

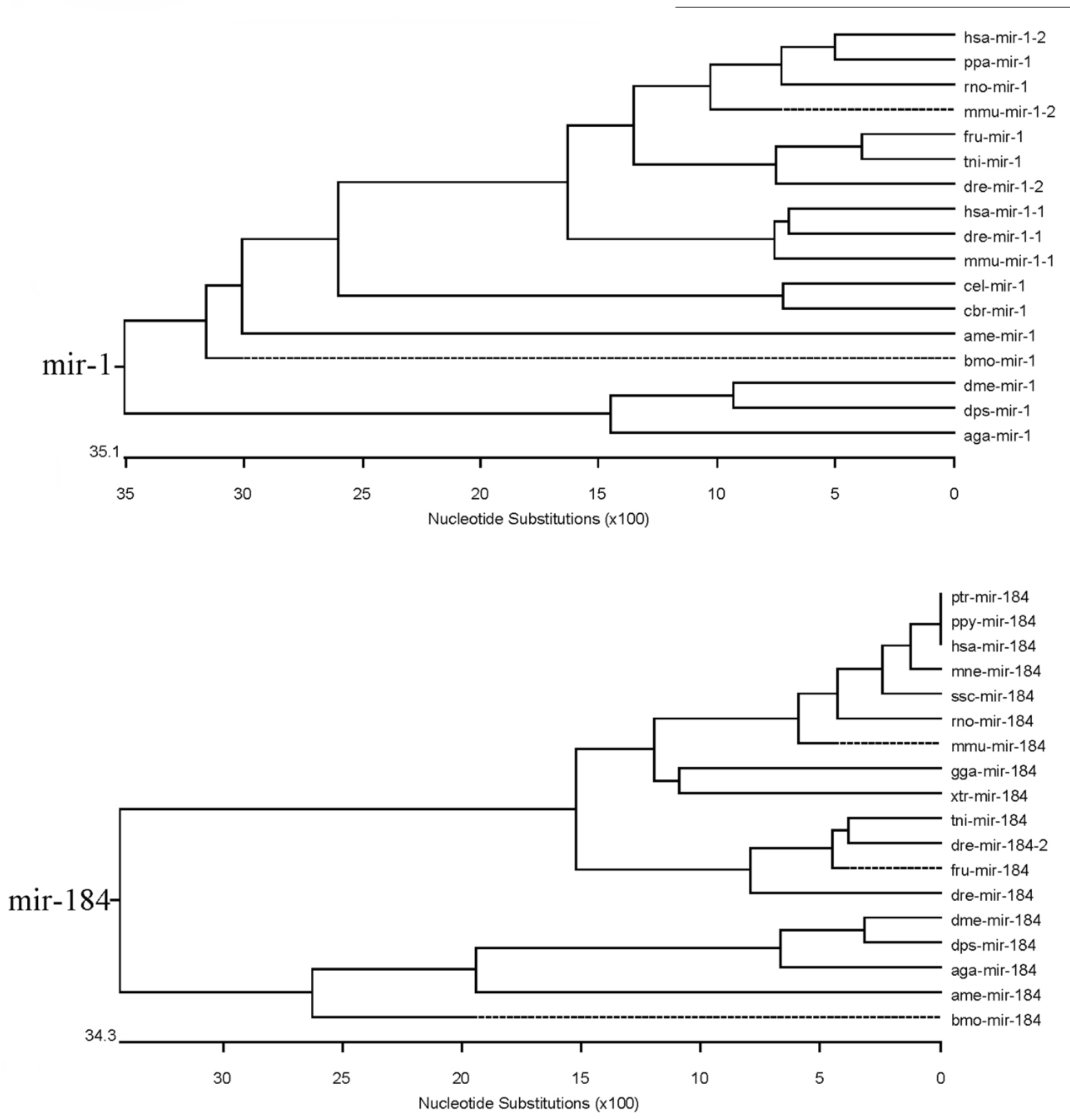

Figure 6

Phylogenetic tree of the mir-I family and the mir-I84 family.

tings for base pairing of binding sites (see Materials and Methods) based on this knowledge. Applying these settings, many targets predicted by functional conservation, such as B. mori homologs of HLHmdelta and M4, would not have been predicted because of stringent filtering of the settings for miRNA-mRNA duplexes. However, using the settings, the predicted binding sites of targets can bind perfectly to miRNA, and the target sites belong mainly to the three categories of known miRNA target sites classified by Sethupathy et al. (2006)[49] [see Additional file 3]. Nevertheless, we identified too many potential binding sites to test experimentally. According to a previous suggestion [49], genes with multiple predicted target sites (61 genes here accounting for $11 \%$ of all predicted targets) 


\section{bmo-mir-1}

\begin{tabular}{|c|c|c|c|c|c|}
\hline & $u$ & a & $c$ & uuc: & gucau \\
\hline$g c c u$ & $g c g c a$ & guuecgugeuuc & uuac & ccaua & u \\
\hline | | | | & 1111 & 11111111111 & | | | | & | | | | | & $g$ \\
\hline cggg & cgcgu & cgagguaugaag & aaug & gguau & u \\
\hline & - & - & a & uaa & acuaa \\
\hline
\end{tabular}

\section{aga-mir-1}

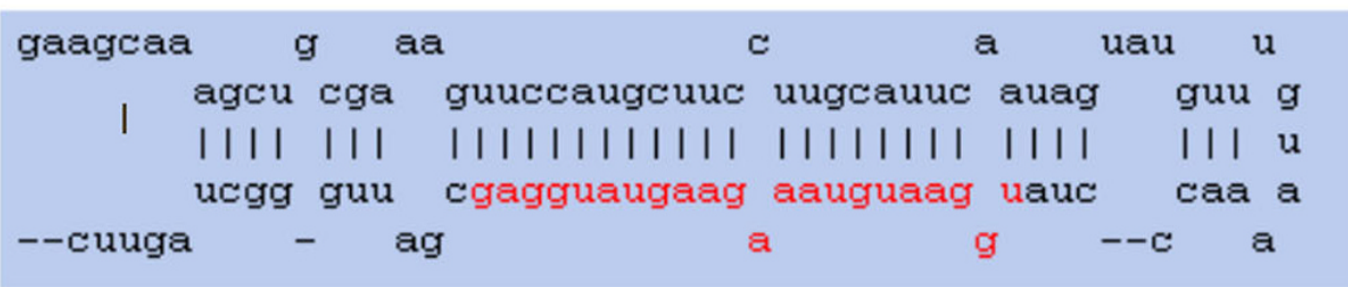

\section{ame-mir-1}

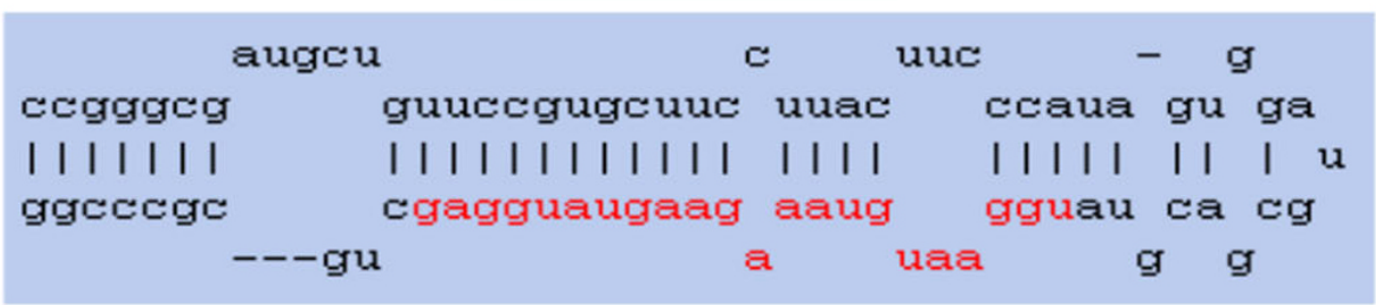

\section{dme-mir-1}

\begin{tabular}{|c|c|c|c|c|c|c|}
\hline uuca & uuu & $--g a$ & $\mathrm{c}$ & a & - & $a u$ \\
\hline$g c c$ & ga & guuccaugcuuc & uugcauuc & aua & guu & a \\
\hline I I | & | | & |||||||||||| & | I | | | | & | | | & | I | & u \\
\hline $\operatorname{cgg}$ & $\mathrm{cu}$ & cgagguaugaag & aauguaag & uau & cga & $\mathrm{u}$ \\
\hline -gag & $--u$ & aaag & a & $g$ & a & $a c$ \\
\hline
\end{tabular}

\section{dps-mir-1}

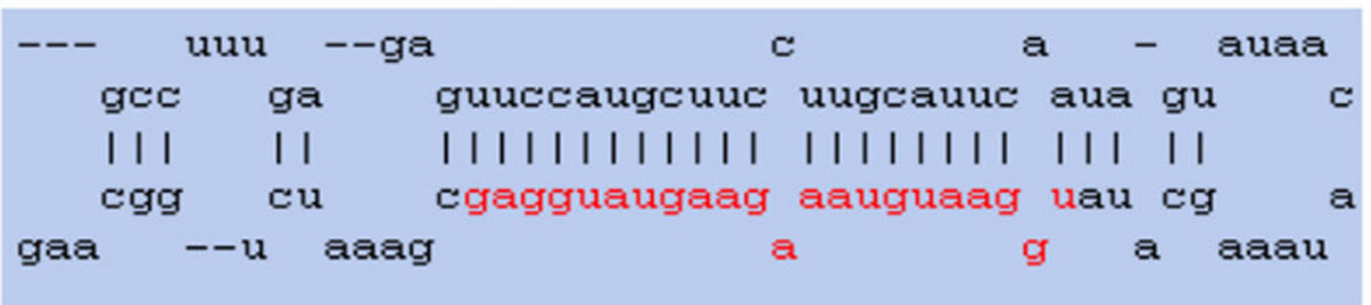

Figure 7

Comparison of the secondary structures of bmo-miR-I, aga-miR-I, ame-miR-I, dme-miR-I and dps-miR-I. 
should be considered strong candidates for future functional studies.

\section{Conclusion}

We identified $46 \mathrm{~B}$. mori miRNAs, 13 of which were miRNA*s, by combining computational predictions with microarray assays. We identified a novel small RNA and 21 plausible B. mori miRNAs that could not be found in the available $B$. mori genome, but which were detected by microarray. The small novel RNA, bmo-miR-100-like, was identified using the known miRNA aga-miR-100 as a probe; it was detected by microarray assay and Northern blotting, but its precursor sequences failed to fold into a hairpin structure. Northern blotting revealed that some $B$. mori miRNA genes were expressed only during specific stages, indicating that $B$. mori miRNA genes have developmentally regulated expression patterns. For example, bmomiR-277 was expressed only during the moth stage. We identified two miRNA gene clusters in the B. mori genome, and $b m o-m i R-2 b$ was identified as a new member of the mir-2 family. We found that methylation of the DNA probe used to detect the miRNA by Northern blotting may increase its sensitivity. According to functional conservation in various species, we predicted that 11 miRNAs may regulate $13 \mathrm{~B}$. mori orthologs of the 25 known Drosophila miRNA-targeted genes. A total of 547 targeted genes were predicted from $1671 \mathrm{~B}$. mori genes according to the target sites predicted by settings hybrid 11 and hybridf22. From the predicted genes, 61 genes each contained multiple predicted target sites and could be candidates for the functional study of miRNA. Three GO terms, "binding", "catalytic activity" and "physiological process", were overrepresented for the predicted genes.

Identification of putative miRNAs in other organisms is a worthwhile approach for increasing the understanding of the roles of miRNA. Identification of miRNAs in B. mori may help to elucidate the mechanisms underlying simple and complex regulation of the 42-day lifecycle of B. mori, which consists of six periods of ecdysis and four periods of metamorphosis.

\section{Methods}

miRNA reference sets and the $B$. mori genome

Prediction and identification of miRNAs in B. mori is shown in Figure 8. We downloaded 78 miRNAs from $D$. melanogaster, 73 from D. pseudoobscura, 25 from A. mellifera, 38 from A. gambiae, and their precursor sequences from MicroRNA Registry release 7.1 [21,22,55]. These sequences were used as a reference set to predict miRNA in B. mori. Genomic sequences for B. mori were downloaded from the Beijing Genomics Institute $[25,26,56]$. The total size of $B$. mori genome is $428.7 \mathrm{Mb}$, containing 23,156 scaffolds and 66,482 contigs.

\section{Prediction of B. mori miRNAs by homology search}

We used the sequences of known miRNAs (vide supra) and pre-miRNAs in the reference set as query sequences for BLAST searches against the $B$. mori genome; we used a word size of seven and an E-value cutoff of ten.

Four criteria were used to predict $B$. mori miRNAs and premiRNAs according to BLAST search results: (1) there was $>80 \%$ similarity between each potential B. mori miRNA and the corresponding miRNA in the reference set; $(2)$ the difference in the lengths between each potential $B$. mori pre-miRNA and the corresponding pre-miRNA in reference set was $<5-\mathrm{nt}$, and the corresponding positions of the mature miRNAs in their pre-miRNAs were nearly identical; (3) the lowest free energy for folding of the secondary structures for each potential B. mori pre-miRNA, as predicted by the mfold program [37], was less than -20 kcal/ mol; and (4) the secondary structures of the predicted premiRNA satisfied the requirements set out in reference [23], i.e., a potential fold-back precursor structure must contain the 22 -nt miRNA sequence within one arm of the hairpin, and the hairpin must include at least $16 \mathrm{bp}$ within the first $22 \mathrm{nt}$ of the miRNA. The structure should not contain large internal loops or bulges, particularly not large asymmetric bulges ( $<5-\mathrm{nt})$.

\section{Paraflo $^{\mathrm{TM}}$ miRNA microarray assay}

Based on the predicted results and the sequences of known miRNAs in Arthropoda, Nematoda, and human, we designed probes to detect $B$. mori miRNAs in $B$. mori pupa and moth stages by microarray profiling.

Microarray assays were done using a service provider (LC Sciences, Houston, USA). The assay was done on $5-\mu \mathrm{g}$ total RNA samples from B. mori pupa (labeled cy3) and moth (labeled cy5), which were size fractionated using a mirVana Isolation kit (Ambion, Austin, USA); the small RNAs (<200 nt) were 3'-extended with a poly(A) tail using poly(A) polymerase. An oligonucleotide tag was then ligated to the poly(A) tail for later staining with fluorescent dye; two different tags were used for the two RNA samples in dual-sample experiments. Hybridization was done overnight on a $\mu$ ParaFlo microfluidic chip using a micro-circulation pump (Atactic Technologies, Houston, USA) [57]. On the microfluidic chip, each detection probe consisted of a chemically modified nucleotide coding segment complementary to the target miRNA (miRBase, http://microrna.sanger.ac.uk/ sequences/) or other RNA (control or our defined sequences) and a spacer segment of polyethylene glycol to extend the coding segment away from the substrate. Detection probes were made by in situ synthesis using photogenerated reagent (PGR) chemistry. The hybridization melting temperatures were balanced by chemical modifications of the detection probes. Hybridizations used $100 \mathrm{~L} 6 \times$ SSPE buffer $\left(0.90 \mathrm{M} \mathrm{NaCl}, 60 \mathrm{mM} \mathrm{Na}_{2} \mathrm{HPO}_{4}, 6 \mathrm{mM}\right.$ EDTA, pH 


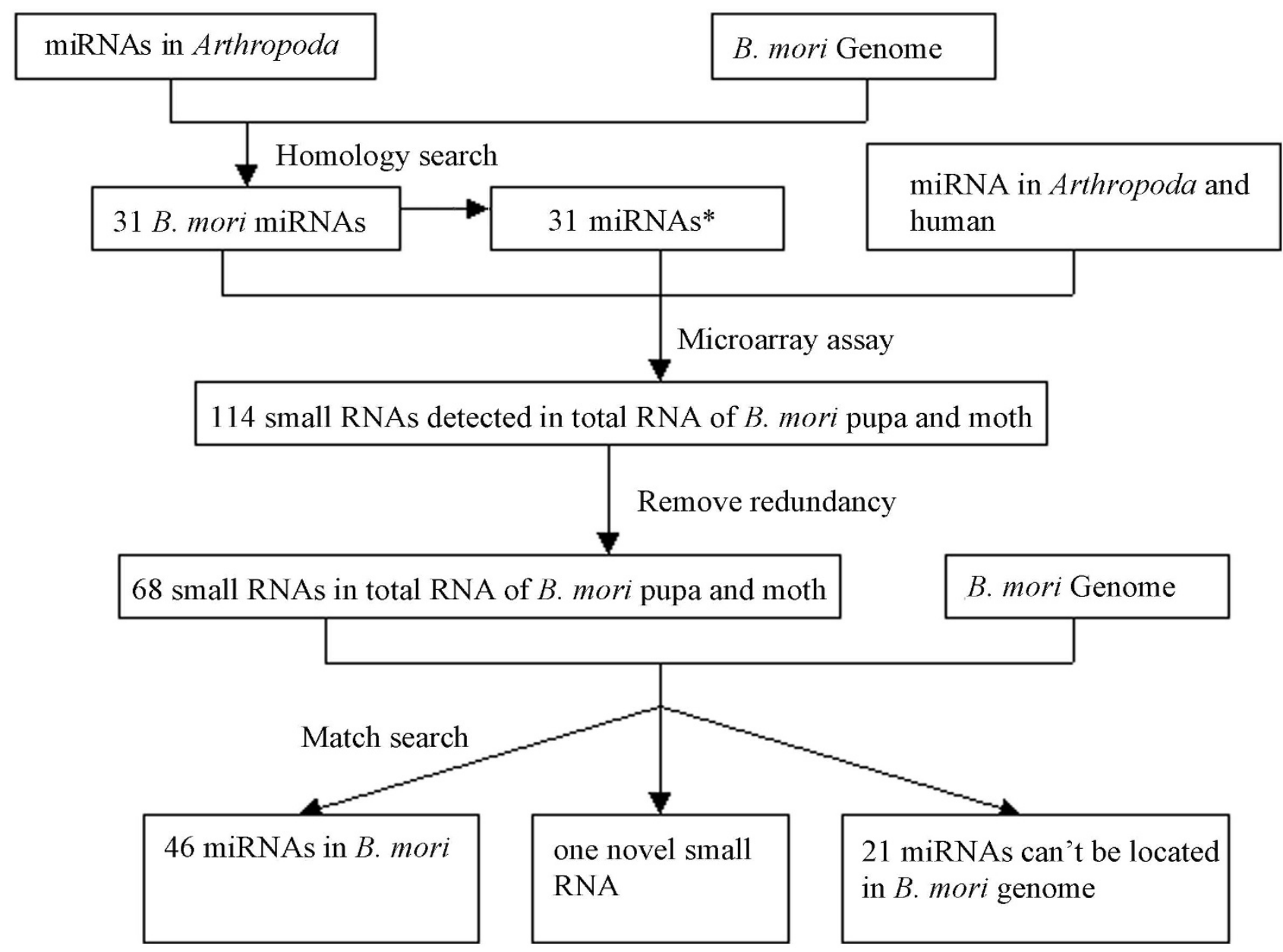

\section{Figure 8}

Predictions and microarray assays for miRNA in B. mori.

6.8) containing $25 \%$ formamide at $34^{\circ} \mathrm{C}$. After hybridization, signals were detected using fluorescence labeling with tag-specfic Cy5 (for B. mori pupa and moth) dyes (Invitrogen, Carlsbad, USA). Hybridization images were collected using a laser scanner (GenePix 4000B, Molecular Device, Sunnyvale, USA) and quantified. Data were analyzed by first subtracting the background, and then normalized using a cyclic LOWESS filter (locally-weighted regression) [58]. For two color experiments, the ratio of the two sets of detected signals ( $\log 2$ transformed, balanced) and p-values of the $t$-test were calculated; differentially detected signals were those with $\mathrm{p}$-values of $<0.01$. Data classification involved a hierarchical clustering method using average linkage and Euclidean distance metric, and was visualized with TIGR's MeV (Multiple Experimental Viewer; Institute for Genomic Research).

\section{Northern blotting}

Total small RNA was isolated from the larva, pupa, and moth stages of the silkworm using mirVana ${ }^{\mathrm{TM}}$ miRNA Isolation Kit (Ambion) according to manufacturer instructions.
Liquid nitrogen was used to freeze samples before RNA extraction. Small RNA was quantified by spectrophotometer (NanoDrop, Wilmington, USA). Twenty micrograms of small RNA was loaded per lane, and resolved on a denaturing $15 \%$ polyacrylamide gel containing $8 \mathrm{M}$ urea at $20 \mathrm{~mA}$; small RNA was transferred by electrophoresis from the gel to a positively charged nylon membrane (Millipore, Shanghai, China) by a semi-dry transfer apparatus (ATTA) at 200 $\mathrm{mA}$ for $2 \mathrm{~h}$. After electroblotting, membranes were ultraviolet crosslinked $(120 \mathrm{~mJ}, 30 \mathrm{~s})$ and baked for $1 \mathrm{~h}$ at $80^{\circ} \mathrm{C}$. DNA probes complementary to small RNA sequences were $5^{\prime}$ end-labeled with $\gamma^{-32}$ P-ATP using a 5' DNA Labeling Kit (Shenergy Biocolor, Shanghai, China). The membrane was prehybridized in prehybridization solution $(6 \times$ SSC, $10 \times$ Denhardt's solution, $0.2 \%$ SDS) at $64^{\circ} \mathrm{C}$ for $1 \mathrm{~h}$. Membranes were hybridized in hybridization solution $(6 \times$ SSC, $5 \times$ Denhardt's solution, $0.2 \%$ SDS) containing $1-5 \times 10^{6}$ cpm 5' end-labeled antisense probes for 18-24 h with gentle agitation at $28^{\circ} \mathrm{C}$. Blots were washed three times for 5 min each at $28^{\circ} \mathrm{C}$ with $6 \times$ SSC and $0.2 \%$ SDS, and once at $42^{\circ} \mathrm{C}$ for $10 \mathrm{~min}$. After the final wash, blots were wrapped 
in plastic wrap and exposed to a phosphorimager screen (Amersham, Piscataway, USA) according to manufacturer instructions.

\section{Computational prediction of miRNA targets}

Functions of the identified B. mori miRNAs were analyzed according to the functional conservation and the potential binding sites between miRNAs and the 3'UTR of responding mRNAs.

Based on the Tarbase database [59], we searched for the known targeted genes of Drosophila orthologs of identified miRNAs. Their B. mori homologs and their 3'UTR were subsequently obtained by the BLAST program. The B. mori miRNA targeted genes were predicted according to the binding between miRNAs and their complementary sites on the 3'UTR of the B. mori homologs by the RNAhybrid program [60].

In general, base pairing between miRNA and the complementary site on mRNA may provide a function prediction for miRNA. We calculated the potential binding sites between identified miRNAs and the 3'UTR of mRNAs of $B$. mori genes using the RNAhybrid program [60]; we used two settings to obtain specific binding sites. The first setting (P-value cutoff of 0.05, minimum free energy cutoff of $-20.0 \mathrm{kcal} / \mathrm{mol}$, maximum internal and bulge loop size of 1) was termed "hybrid11"; if maximum internal and bulge loop size was 2 but the seed region of miRNA could perfectly bind to 3'UTR of a gene (helix constraint of 2.7), we also considered this gene to be a potential miRNA-targeted gene (this setting was termed "hybridf22"). The functional annotation for predicted targeted genes were analyzed using Gene Ontology analysis [61]. B. mori genes were obtained from B. mori Genes Database constructed by us, and which includes 1909 genes. From these genes, 1671 3'UTRs were obtained and used for predicting potential targeted genes.

\section{Authors' contributions}

PAH carried out the prediction and analysis of the miRNA and drafted the manuscript. ZMN performed the experiments, and carried out the analysis of miRNA and drafted the manuscript. JQC, JC, ZBL, QS and LYK performed the experiments. SPZ and XLG helped to analyze the data and draft the manuscript. XFW directed all the work of the manuscript. YFJ and YZZ conceived, and carried out the design and draft of the manuscript. All authors read and approved the final manuscript.

\section{Additional material}

\section{Additional file 1}

Northern blotting analysis of $\mathrm{B}$. mori miRNAs. The data provided show the results of Northern blotting analysis for miRNAs. One table and three figures are in the file. The table shows the 21 plausible miRNAs identified by Northern blotting. Figure 1 displayed the result of Northern blotting analysis for bmo-miR-277. Figure 2 shows improvement of the detectable level of miRNA using methylated probes. Figure 3 shows the multi-alignment of the members of miRNA let-7 family.

Click here for file

[http://www.biomedcentral.com/content/supplementary/1471-

2164-9-248-S1.doc]

\section{Additional file 2}

Functional analysis of miRNAs in B. mori according to functional conservation between silkworm and fruit fly. The data provided show the detailed information regarding predicting targets of miRNAs in $\mathrm{B}$. mori. Targets were predicted according to the binding between miRNAs and $\mathrm{B}$. mori orthologs of known miRNA targets of fruit fly.

Click here for file

[http://www.biomedcentral.com/content/supplementary/1471-

2164-9-248-S2.doc]

\section{Additional file 3}

Functional analysis of miRNAs in B. mori according to binding sites between miRNAs and mRNAs. The data provided show the detailed information for miRNA-targeted genes predicted according to binding sites on $3^{\prime}$ 'UTR of mRNAs. There are six sheets in the EXCEL file, including hybrid11, hybridf22, combination with no redundancy, statistics of binding sites, Go analysis, and binding sites of profilin. Sheet hybrid11 shows detailed information of predicted target genes using setting hybrid11; sheet hybridf 22 shows detailed information of predicted target genes using setting hybridf22; sheet 3, named "combination with no redundancy", shows the results for the sheet hybrid11 and hybridf22 after removing redundancy; sheet 4, named "statistics of binding sites", shows the results of statistic analysis for all genes that were found to have more than one binding site; sheet 5, named "Go analysis", shows the results of Go anal$y$ sis of predicted targeted genes; and sheet 6 , named "binding sites of profilin", shows information of the potential binding sites between the B. mori profilin gene and miRNAs.

Click here for file

[http://www.biomedcentral.com/content/supplementary/14712164-9-248-S3.xls]

\section{Additional file 4}

Phylogeny trees for each of miRNA families. The data provided show the phylogeny trees for each of miRNA families in $\mathrm{B}$. mori.

Click here for file

[http://www.biomedcentral.com/content/supplementary/14712164-9-248-S4.doc]

\section{Additional file 5}

Detailed information of all the 46 identified miRNAs in $\mathrm{B}$. mori. The data provided show the detailed information of all the 46 identified miRNAs in $\mathrm{B}$. mori, including sequences of the pre-miRNA and mature miRNA, the complementary region of miRNAs, the secondary structure of pre-miRNA, and the minimum energy.

Click here for file

[http://www.biomedcentral.com/content/supplementary/14712164-9-248-S5.doc] 


\section{Acknowledgements}

This work was supported by financial grants from the National Basic Research Program of China (973 Program, No.2005CBI21006), the National High Technology Research and Development Program (No. 2007AA02 1703), the National Natural Science Foundation of China (No.307400 I5, No.30670447) and the Zhejiang Natural Science Foundation (No. Y205449, Y304 I22). We thank International Science Editing for English editing.

\section{References}

I. Ambros V: MicroRNA pathways in flies and worms: growth, death, fat, stress, and timing. Cell 2003, I I3(6):673-676.

2. Ambros V: The functions of animal microRNAs. Nature 2004, 43 I (7006):350-355.

3. Lim LP, Lau NC, Weinstein EG, Abdelhakim A, Yekta S, Rhoades MW, Burge CB, Bartel DP: The microRNAs of Caenorhabditis elegans. Genes Dev 2003, I 7(8):991-1008.

4. Carrington JC, Ambros V: Role of microRNAs in plant and animal development. Science 2003, 30 I(563I):336-338.

5. Lee RC, Feinbaum RL, Ambros V: The C. elegans heterochronic gene lin-4 encodes small RNAs with antisense complementarity to lin-14. Cell 1993, 75(5):843-854.

6. Reinhart BJ, Slack FJ, Basson M, Pasquinelli AE, Bettinger JC, Rougvie AE, Horvitz HR, Ruvkun G: The 2 I-nucleotide let-7 RNA regulates developmental timing in Caenorhabditis elegans. Nature 2000, 403(6772):901-906.

7. Lagos-Quintana M, Rauhut R, Lendeckel W, Tuschl T: Identification of novel genes coding for small expressed RNAs. Science 200 I, 294(5543):853-858.

8. Lau NC, Lim LP, Weinstein EG, Bartel DP: An abundant class of tiny RNAs with probable regulatory roles in Caenorhabditis elegans. Science 200I, 294(5543):858-862.

9. Lee RC, Ambros V: An extensive class of small RNAs in Caenorhabditis elegans. Science 200I, 294(5543):862-864.

10. Grad Y, Aach J, Hayes GD, Reinhart BJ, Church GM, Ruvkun G, Kim $\mathrm{J}$ : Computational and experimental identification of $\mathbf{C}$. elegans microRNAs. Mol Cell 2003, I I(5): I 253-I 263.

II. Lai EC, Tomancak P, Williams RW, Rubin GM: Computational identification of Drosophila microRNA genes. Genome Biol 2003, 4(7):R42.

12. Wang XJ, Reyes JL, Chua NH, Gaasterland T: Prediction and identification of Arabidopsis thaliana microRNAs and their mRNA targets. Genome Biol 2004, 5(9):R65

13. Jones-Rhoades MW, Bartel DP: Computational identification of plant microRNAs and their targets, including a stressinduced miRNA. Mol Cell 2004, I 4(6):787-799.

14. Bonnet E, Wuyts J, Rouze P, Peer Y Van de: Evidence that microRNA precursors, unlike other non-coding RNAs, have lower folding free energies than random sequences. Bioinformatics 2004, 20(I7):291।-2917.

15. Yoon S, De Micheli G: Prediction of regulatory modules comprising microRNAs and target genes. Bioinformatics 2005, 2 I(Suppl 2):ii93-I00.

16. Wang X, Zhang J, Li F, Gu J, He T, Zhang X, Li Y: MicroRNA identification based on sequence and structure alignment. Bioinformatics 2005, 2 I ( I 8):36 I 0-36 I4.

17. Nam JW, Shin KR, Han J, Lee Y, Kim VN, Zhang BT: Human microRNA prediction through a probabilistic co-learning model of sequence and structure. Nucleic Acids Res 2005, 33(I I):3570-358I.

18. Dezulian T, Remmert M, Palatnik JF, Weigel D, Huson DH: Identification of plant microRNA homologs. Bioinformatics 2006, 22(3):359-360.

19. Tong CZ, Jin YF, Zhang YZ: Computational prediction of microRNA genes in silkworm genome. J Zhejiang Univ Sci B 2006, 7(10):806-8I6.

20. Helvik SA, Snove O Jr, Saetrom P: Reliable prediction of Drosha processing sites improves microRNA gene prediction. Bioinformatics 2007, 23(2): |42-| 49.

21. Griffiths-Jones S, Grocock RJ, van Dongen S, Bateman A, Enright AJ: miRBase: microRNA sequences, targets and gene nomenclature. Nucleic Acids Res 2006:DI40-I44.

22. Griffiths-Jones S: The microRNA Registry. Nucleic Acids Res 2004:DI09-III.
23. Ambros V, Bartel B, Bartel DP, Burge CB, Carrington JC, Chen X, Dreyfuss G, Eddy SR, Griffiths-Jones S, Marshall M, et al.: A uniform system for microRNA annotation. RNA 2003, 9(3):277-279.

24. Arteaga-Vazquez M, Caballero-Perez J, Vielle-Calzada JP: A family of microRNAs present in plants and animals. Plant Cell 2006, I 8( I 2):3355-3369.

25. Xia Q, Zhou Z, Lu C, Cheng D, Dai F, Li B, Zhao P, Zha X, Cheng T, Chai $C$, et al:: $A$ draft sequence for the genome of the domesticated silkworm (Bombyx mori). Science 2004, 306(5703): 1937-1940.

26. Wang J, Xia Q, He X, Dai M, Ruan J, Chen J, Yu G, Yuan H, Hu Y, Li $\mathrm{R}$, et al.: SilkDB: a knowledgebase for silkworm biology and genomics. Nucleic Acids Res 2005:D399-402.

27. Schwarz DS, Hutvagner G, Du T, Xu Z, Aronin N, Zamore PD: Asymmetry in the assembly of the RNAi enzyme complex. Cell 2003, I I5(2): 199-208.

28. Gregory RI, Chendrimada TP, Cooch N, Shiekhattar R: Human RISC couples microRNA biogenesis and posttranscriptional gene silencing. Cell 2005, I 23(4):63 I-640.

29. Velkey JM, Slawny NA, Gratsch TE, O'Shea KS: Gene silencing using RNA interference in embryonic stem cells. Methods $\mathrm{Mol}$ Biol 2006, 329:233-26I.

30. Bartel DP: MicroRNAs: genomics, biogenesis, mechanism, and function. Cell 2004, I I 6(2):28I-297.

31. Rana TM: Illuminating the silence: understanding the structure and function of small RNAs. Nat Rev Mol Cell Biol 2007, 8(I):23-36.

32. Zhang $B$, Wang $Q, \operatorname{Pan} X$ : MicroRNAs and their regulatory roles in animals and plants. J Cell Physiol 2007, 2 I 0(2):279-289.

33. Behura SK: Insect microRNAs: Structure, function and evolution. Insect Biochem Mol Biol 2007, 37(I):3-9.

34. Denli AM, Tops BB, Plasterk RH, Ketting RF, Hannon GJ: Processing of primary microRNAs by the Microprocessor complex. Nature 2004, 432(7014):231-235.

35. Kloosterman WP, Wienholds E, Ketting RF, Plasterk RH: Substrate requirements for let-7 function in the developing zebrafish embryo. Nucleic Acids Res 2004, 32(2I):6284-6291.

36. Liu S, Xia Q, Zhao P, Cheng T, Hong K, Xiang Z: Characterization and expression patterns of let-7 microRNA in the silkworm (Bombyx mori). BMC Dev Biol 2007, 7:88.

37. Zuker M: Mfold web server for nucleic acid folding and hybridization prediction. Nucleic Acids Res 2003, 3 I (I 3):3406-34I 5.

38. Grun D, Wang YL, Langenberger D, Gunsalus KC, Rajewsky N: microRNA target predictions across seven Drosophila species and comparison to mammalian targets. PLoS Comput Biol 2005, I (I): el 3 .

39. Sethupathy P, Corda B, Hatzigeorgiou AG: TarBase: A comprehensive database of experimentally supported animal microRNA targets. RNA 2006, I 2(2): 192-197.

40. Roder K, Hung MS, Lee TL, Lin TY, Xiao H, Isobe KI, Juang JL, Shen C]: Transcriptional repression by Drosophila methyl-CpGbinding proteins. Mol Cell Biol 2000, 20(19):740 I-7409.

4I. Guimaraes-Sternberg C, Meerson A, Shaked I, Soreq H: MicroRNA modulation of megakaryoblast fate involves cholinergic signaling. Leuk Res 2006, 30(5):583-595.

42. Majlessi M, Nelson NC, Becker MM: Advantages of 2'-O-methyl oligoribonucleotide probes for detecting RNA targets. Nucleic Acids Res 1998, 26(9):2224-2229.

43. Tanzer A, Stadler PF: Molecular evolution of a microRNA cluster. J Mol Biol 2004, 339(2):327-335.

44. Aravin AA, Lagos-Quintana M, Yalcin A, Zavolan M, Marks D, Snyder $B$, Gaasterland T, Meyer J, Tuschl T: The small RNA profile during Drosophila melanogaster development. Dev Cell 2003, 5(2):337-350.

45. Seitz H, Royo H, Bortolin ML, Lin SP, Ferguson-Smith AC, Cavaille J: A large imprinted microRNA gene cluster at the mouse DlkI-GtI2 domain. Genome Res 2004, | 4(9): | 74|-1748.

46. Valencia-Sanchez MA, Liu J, Hannon GJ, Parker R: Control of translation and mRNA degradation by miRNAs and siRNAs. Genes Dev 2006, 20(5):5I5-524.

47. Lee $Y$, Jeon K, Lee JT, Kim S, Kim VN: MicroRNA maturation: stepwise processing and subcellular localization. EMBO J 2002, 2 I ( I 7):4663-4670.

48. Stark A, Brennecke J, Russell RB, Cohen SM: Identification of Drosophila MicroRNA targets. PLOS Biol 2003, I (3):E60. 
49. Sethupathy P, Megraw M, Hatzigeorgiou AG: A guide through present computational approaches for the identification of mammalian microRNA targets. Nat Methods 2006, 3(I I):88I-886.

50. Joglekar MV, Parekh VS, Mehta S, Bhonde RR, Hardikar AA: MicroRNA profiling of developing and regenerating pancreas reveal post-transcriptional regulation of neurogenin3. Dev Biol 2007, 31 I (2):603-612.

5I. Asangani IA, Rasheed SA, Nikolova DA, Leupold JH, Colburn NH, Post S, Allgayer H: MicroRNA-2I (miR-2I) post-transcriptionally downregulates tumor suppressor Pdcd4 and stimulates invasion, intravasation and metastasis in colorectal cancer. Oncogene 2007.

52. Weaver DB, Anzola JM, Evans JD, Reid JG, Reese JT, Childs KL, Zdobnov EM, Samanta MP, Miller J, Elsik CG: Computational and transcriptional evidence for microRNAs in the honey bee genome. Genome Biol 2007, 8(6):R97.

53. Lai EC: Micro RNAs are complementary to 3' UTR sequence motifs that mediate negative post-transcriptional regulation. Nat Genet 2002, 30(4):363-364.

54. Clarke SR, Staiger CJ, Gibbon BC, Franklin-Tong VE: A potential signaling role for profilin in pollen of Papaver rhoeas. Plant Cell 1998, I0(6):967-979.

55. miRBase website [http://microrna.sanger.ac.uk/sequences/
(n) index.shtml]

56. Beijing Genomics Institute website [http://silkworm.genom ics.org.cn/]

57. Gao X, Gulari E, Zhou X: In situ synthesis of oligonucleotide microarrays. Biopolymers 2004, 73(5):579-596.

58. Bolstad BM, Irizarry RA, Astrand M, Speed TP: A comparison of normalization methods for high density oligonucleotide array data based on variance and bias. Bioinformatics 2003, 19(2): 185-193.

59. Tarbase website [http://www.diana.pcbi.upenn.edu/tarbase.html]

60. Rehmsmeier M, Steffen P, Hochsmann M, Giegerich R: Fast and effective prediction of microRNA/target duplexes. RNA 2004, IO(10): I507-1517.

61. Gene Ontology analysis website [http://goblet.molgen.mpg.de/ cgi-bin/goblet/webapp-goblet.cgi?menu=Form]

Publish with Bio Med Central and every scientist can read your work free of charge

"BioMed Central will be the most significant development for disseminating the results of biomedical research in our lifetime. "

Sir Paul Nurse, Cancer Research UK

Your research papers will be:

- available free of charge to the entire biomedical community

- peer reviewed and published immediately upon acceptance

- cited in PubMed and archived on PubMed Central

- yours - you keep the copyright
BioMedcentral 